\title{
A multi-shRNA vector enhances the silencing efficiency of exogenous and endogenous genes in human cells
}

\author{
YANJIE WENG $^{1}$, YING SHI ${ }^{1}$, XI XIA ${ }^{1,2}$, WENJUAN ZHOU ${ }^{1}$, HONGYAN WANG $^{1}$ and CHANGYU WANG $^{1}$ \\ ${ }^{1}$ Cancer Biology Research Center, Tongji Hospital, Tongji Medical College, Huazhong University of Science and Technology, \\ Wuhan, Hubei 430030; ${ }^{2}$ Department of Gynecology and Obstetrics, Affiliated Shenzhen Nanshan Hospital, \\ Guangdong Medical College, Shenzhen, Guangdong 518052, P.R. China
}

Received April 23, 2015; Accepted September 27, 2016

DOI: $10.3892 / \mathrm{ol} .2017 .5672$

\begin{abstract}
RNA interference (RNAi) is a powerful technology for suppressing gene function. In most studies, small interfering RNAs (siRNAs) consist of one short hairpin RNA (shRNA) and, therefore, are often unable to achieve loss-of-function of their target genes. In the current study, an RNAi vector containing three shRNAs under the control of three RNA polymerase III U6 promoters was constructed. RNAi vectors containing one or two shRNAs were generated for comparisons. A pilot study targeting exogenously expressed DsRed in the HEK293 cell line revealed promising effects and a high selectivity for the multi-shRNA RNAi vector. Akt2 is constitutively expressed in cultured SKOV3 human ovarian cancer cells, and the multi-shRNA RNAi vector showed a strong efficiency for downregulating the expression of Akt 2 in these cells, with no apparent interferon response. In addition, the Akt2-3shRNA vector, containing three shRNAs targeting Akt2, showed the best effect of all the shRNA vectors in reversing paclitaxel-induced resistance in SKOV3 cells. This study developed a widely applicable resource for enhancing the efficiency of gene silencing and a novel technique for performing complex loss-of-function screens in mammalian cells.
\end{abstract}

\section{Introduction}

RNA interference (RNAi) based on small interfering RNA (siRNA) has been widely exploited for the suppression of gene expression in model organisms such as Caenorhabditis elegans and Drosophila melanogaster (1), as well as in mammalian cells (2). Furthermore, RNAi technology based on short hairpin RNAs (shRNAs) has been widely used in recent years. As compared with shRNAs, siRNAs are easy to handle and require less time to synthesize (3). Although Shan et al (4)

Correspondence to: Dr Changyu Wang, Cancer Biology Research Center, Tongji Hospital, Tongji Medical College, Huazhong University of Science and Technology, 1095 Liberation Avenue, Wuhan, Hubei 430030, P.R. China

E-mail: tjwcy66@163.com

Key words: multi-site short hairpin RNA vector, Akt2, DsRed used small molecules containing different concentrations of siRNA to enhance RNAi, mammalian cells are often insensitive to the transfection methods used to introduce synthesized siRNAs into cells (5). Furthermore, the effects of synthesized siRNAs are transient and do not allow for stable inhibition of a specific gene (6).

Therefore, an alternative approach for introducing plasmids or viruses carrying shRNAs into mammalian cells for large-scale, loss-of-function screens is required. Furthermore, this approach should achieve stable and highly effective gene suppression in a variety of mammalian cell types. However, the gene inhibitory effects are limited by the variable efficiencies and specificities of the empirically designed siRNA or shRNA constructs (7). Previous studies have screened for the optimum shRNA construct and have demonstrated that some siRNAs have 'off-target' effects, such as interfering with the expression or function of other genes or proteins (8-10). To circumvent these limitations, several different sequence fragments of a target gene were introduced into a cell concurrently, which resulted in an enhanced silencing efficiency when attempting to inhibit the function of a single gene (11).

Lentiviral shRNA vectors are currently the most appealing tool for the efficient delivery and stable suppression of genes in nearly all cell types, and many researchers have used this approach to achieve highly effective gene suppression in mammalian cells $(5,12-14)$. However, this technique has inherent limitations associated with the use of lentiviruses; in particular, it is unsafe for researchers to be recurrently exposed to lentiviruses, especially if the laboratory is not equipped with a biological safety cabinet. Therefore, the present study selected the enhanced green fluorescent protein (EGFP)-C1 plasmid (pEGFP-C1) for the construction of multi-shRNA RNAi vectors to provide an effective and safe method for introducing shRNA into cells.

The authors of the present study have performed several studies using shRNA plasmid vectors. In our previous study, two shRNA interference vectors were used to silence one gene, and it was demonstrated that this method had better silencing effects than when a single shRNA vector was used (15). In another study, a multi-shRNA vector was constructed to circumvent the challenges associated with transfecting some human cancer cells with more than one vector (16). This vector contained three U6 promoters and at least three shRNA fragments that were able to 
simultaneously inhibit one gene at multiple sites. Based on this idea, a multi-shRNA vector platform able to direct the synthesis of shRNAs in human cells was developed in the present study.

The current study also addressed the silencing efficiency of endogenous and exogenous genes. First, the construction strategy of multi-shRNA vectors was described. Second, the silencing effects of the multi-shRNA vector on an exogenously expressed gene (DsRed) in HEK293 cells were compared to those of vectors containing one and two shRNAs. Third, the potential off-target effects of these RNAi processes, which may occur when dsRNAs that are longer than $30 \mathrm{bp}$ are introduced into mammalian cells $(17,18)$, were tested. Finally, the silencing and off-target effects of the multi-shRNA vector targeting the Akt2 gene in SKOV3 human ovarian cancer cells were verified and compared with vectors containing one or two shRNAs targeting the same gene. In addition, the viability and apoptosis rate of SKOV3 cells treated with paclitaxel (PTX) were evaluated $48 \mathrm{~h}$ after silencing of the Akt2 gene using the various shRNA vectors.

The results of the present study suggested that the multi-shRNA vector was more effective at gene suppression than the single- or double-assembled shRNA vectors. Therefore, the technique of multiple shRNA vector construction may be applied to RNAi library generation and loss-of-function studies in mammalian cells.

\section{Materials and methods}

Plasmid construction. The pEGFP-C1-U6 vector, which encodes three human U6 shRNA promoters, was constructed at our laboratory and was based on the pEGFP-C1 vector from Promega Corporation (Madison, WI, USA). The pSIREN-DNR-DSRed-Express vector was purchased from BD Biosciences (Franklin Lakes, NJ, USA).

Three target sites for silencing the DsRed gene were designed by referring to the technical information provided by Ambion (Thermo Fisher Scientific, Inc., Waltham, MA, USA). These sequences were as follows: 5'-AAGGAGTTCATGCGC TTCAAG-3' (25-46 bp), 5'-AAGTTCATCGGCGTGAAC TTC-3' (367-388 bp) and 5'-AAGTCCATCTACATGGCC AAG-3' (532-553 bp) (Fig. 1A). To prevent interference with the expression of off-target genes, a Basic Local Alignment Search Tool (http://blast.ncbi.nlm.nih.gov/Blast.cgi) analysis was performed to confirm that the selected oligonucleotide sets showed no homology with any other genes.

By ligating three fragments of annealed, forward and reverse oligonucleotides encoding shRNA sequences into the pEGFPC1-U6 vector (one after each U6 promoter), the multi-shRNA vector was constructed (Fig. 1B). The sequences of the shRNAs targeting DsRed are shown in Table I. The annealed shRNAs were ligated into the Bam HI and Pst I (shRNA1), Sac I and Kpn I (shRNA2) or Hind III and Mlu I (shRNA3) sites of the pEGFPC1-U6 vector using the corresponding restriction enzymes. The restriction maps of these shRNAs were provided in our previous study (16). This multiple shRNA-targeting DsRed plasmid was termed DsRed-3shRNA.

The production strategy for the vectors containing single shRNAs is shown in Fig. 1C. These vectors were designated DsRedshRNA1, DsRedshRNA2 and DsRedshRNA3. The shRNA fragments containing one human U6 promoter and one target site were obtained in vitro using polymerase chain reaction (PCR) and the QIAquick PCR Purification kit (Qiagen GmbH, Hilden, Germany); each PCR fragment was $267 \mathrm{bp}$ in length (Fig. 1D). The three fragments were obtained using the same forward primer (5'-GGAATTCAAGCGGCC GCATAACTTCG-3'), but different reverse primers (shown in Table II). The full-length DsRed plasmid (maintained by our lab) was used as the template for PCR, and the PCR products were double-digested with Eco RI and Mlu I and ligated into the Eco RI and Mlu I cloning sites of pEGFP-C1 to form the single-site shRNA vectors. pEGFP-C1 containing a non-specific sequence was used as a control.

Vectors containing the inserted fragments were selected following digestion with Eco RI and Mlu I enzymes and analysis of the plasmids by $1.5 \%$ agarose gel electrophoresis (Fig. 1E and F). Sequencing was performed to confirm the lack of mutations.

Production of the multi-shRNA RNAi vector targeting Akt2, designated Akt2-3shRNA, the two-site shRNA vector, designated Akt2-2shRNA, and the single-site shRNA vector, designated Akt2-shRNA, was described in our previous studies $(15,16)$.

Cell lines and cultures. The HEK293 human embryonic kidney cell line and the SKOV3 human ovarian cancer cell line were purchased from American Type Culture Collection (Manassas, VA, USA). HEK293 cells were maintained in Dulbecco's modified Eagle's medium (DMEM) (Gibco; Thermo Fisher Scientific, Inc.) containing 10\% fetal bovine serum (FBS) (Gibco; Thermo Fisher Scientific, Inc.), and SKOV3 cells were maintained in McCoy's 5A medium (Gibco; Thermo Fisher Scientific, Inc.) supplemented with 20\% FBS, at $37^{\circ} \mathrm{C}$ in a humidified atmosphere containing $5 \% \mathrm{CO}_{2}$. The cells were routinely subcultured twice per week.

Establishment of stable HEK293 cell lines expressing DsRed. HEK 293 cells $\left(1 \times 10^{6} / \mathrm{ml}\right)$ were seeded into 6-well plates in $2 \mathrm{ml}$ DMEM containing $10 \%$ FBS without antibiotics 1 day prior to transfection. Cells at $80 \%$ confluency were transfected with $4 \mu \mathrm{g}$ full-length DsRed plasmid using $5 \mu \mathrm{l}$ Lipofectamine 2000 reagent (Invitrogen; Thermo Fisher Scientific, Inc.) per well, according to the manufacturer's protocol. After $24 \mathrm{~h}$, the cells were split to $30-40 \%$ confluency, and $300 \mu \mathrm{g} / \mathrm{ml}$ hygromycin B (Calbiochem ${ }^{\circledR}$ Small Molecules; Merck Millipore, Darmstadt, Germany) was added to the medium for screening over the following 2 weeks until red-fluorescent monoclones were formed. The red monoclones were isolated, seeded into a 24-well plate and grown until they reached $30 \%$ confluency. These cells were then treated with DMEM containing 10\% FBS and $300 \mu \mathrm{g} / \mathrm{ml}$ hygromycin B for another week. When the red-fluorescent cells were $>90 \%$ confluent, the concentration of hygromycin B in the medium was decreased to $100 \mu \mathrm{g} / \mathrm{ml}$ and the red cells were allowed to grow for another 2 weeks to form the stable HEK293/DsRed cell line.

Transfection of cells. For RNAi, HEK293/DsRed and SKOV3 cells that had been cultured in 6-well plates were transfected with the indicated plasmids using Lipofectamine 2000. After $6 \mathrm{~h}$, the transfection solution was removed and replaced with fresh complete growth medium. The cells were then assayed 
for the expression of the shRNAs at various time points following transfection. In the present study, $2 \mu \mathrm{l}$ exogenous IFN- $\beta$ (Sigma-Aldrich; Merck Millipore) into the medium of 293/DsRed/DsRedshRNA1, 293/DsRed/DsRedshRNA2, 293/DsRed/DsRedshRNA3, 293/DsRed/DsRed-3shRNA and 293/DsRed cells for more than $16 \mathrm{~h}$ at $37^{\circ} \mathrm{C}$ in a humidified atmosphere containing $5 \% \mathrm{CO}_{2}$ to induce the IFN signaling pathways. The cells were seeded onto 6 -well plates at a cell density of $1 \times 10^{6}$ cells/ml per well in $2 \mathrm{ml}$ DMEM with $10 \%$ FCS.

Reverse transcription-quantitative polymerase chain reaction (RT-qPCR) and semiquantitative RT-PCR. Total RNA was extracted from the cells at various time points following transfection using TRIzol reagent (Invitrogen; Thermo Fisher Scientific, Inc.). RT-qPCR was performed using the Stratagene Mx3000P System (Agilent Technologies, Inc., Santa Clara, CA, USA) and the SYBR Green Realtime PCR Master Mix (Toyobo Co., Ltd., Osaka, Japan). All primer combinations that were used are shown in Table III. The primers were produced by Yingjun Biotechnology Corporation (Shanghai, China). The PCR cycling conditions were $95^{\circ} \mathrm{C}$ for $1 \mathrm{~min}$, followed by 40 cycles of $95^{\circ} \mathrm{C}$ for $15 \mathrm{sec}, 60^{\circ} \mathrm{C}$ for $15 \mathrm{sec}$ and $72^{\circ} \mathrm{C}$ for $30 \mathrm{sec}$. GAPDH served as an endogenous control. All reactions were run in triplicate, and the fold-amplification of the genes was determined using the $2^{-\Delta \Delta \mathrm{Cq}}$ method (19).

For the semiquantitative RT-PCR, the cycling conditions were as follows: $94^{\circ} \mathrm{C}$ for $5 \mathrm{~min} ; 30$ cycles at $94^{\circ} \mathrm{C}$ for $30 \mathrm{sec}$, $60^{\circ} \mathrm{C}$ for $30 \mathrm{sec}$ and $72^{\circ} \mathrm{C}$ for $30 \mathrm{sec}$; and $72^{\circ} \mathrm{C}$ for $10 \mathrm{~min}$. PCR products $(10 \mu \mathrm{l})$ were analyzed by $1.5 \%$ agarose gel electrophoresis in the presence of ethidium bromide for UV light transilluminator visualization.

Western blotting. The harvested cells were lysed using lysis buffer (Beyotime Institute of Biotechnology, Haimen, China), and protein lysates $(50 \mu \mathrm{g})$ were denatured in SDS sample buffer at $100^{\circ} \mathrm{C}$ for $10 \mathrm{~min}$. The proteins were then separated by $10 \%$ SDS-PAGE and transferred onto nitrocellulose membranes. The membranes were blocked with $5 \%$ non-fat milk in TBST (25 mmol/1 Tris- $\mathrm{HCl}, \mathrm{pH} 7.5,137 \mathrm{mmol} / \mathrm{l} \mathrm{NaCl}, 2.7 \mathrm{mmol} / \mathrm{l} \mathrm{KCl}$ and $0.05 \%$ Tween-20) for $1 \mathrm{~h}$ at $37^{\circ} \mathrm{C}$, followed by incubation with mouse anti-signal transducer and activator of transcription (STAT1; 1:1,000; cat. no. MA1-037X; NeoMarkers; Thermo Fisher Scientific, Inc.), rabbit anti-Akt2 (1:1,000; cat. no. 3063; Cell Technology, Inc., Danvers, MA, USA) and mouse anti- $\beta$-actin (1:1,000; cat. no. sc-69879; Santa Cruz Biotechnology, Inc., Dallas, TX, USA) primary antibodies overnight at $4^{\circ} \mathrm{C}$. The membrane was washed three times with TBST, followed by incubation with horseradish peroxidase-conjugated goat IgG secondary antibodies (1:1,000; cat. nos. sc-2004 and sc-2005; Santa Cruz Biotechnology, Inc.) for $2 \mathrm{~h}$ at room temperature. The antibodies were visualized using NBT/BCIP/buffer (1:1:50; Roche Diagnostics, Basel, Switzerland). The band intensities were quantified using ImageJ 1.48 software (National Institutes of Health, Bethesda, MD, USA).

Flow cytometric analysis. The DsRed fluorescence intensity and apoptosis of HEK293/DsRed cells was evaluated using flow cytometry on a FACSort ${ }^{\mathrm{TM}}$ flow cytometer (BD Biosciences). The cells were harvested using $0.2 \%$ trypsin, washed twice with PBS and resuspended in PBS at a density of
$1 \times 10^{6}$ cells. For analysis of apoptosis, the cells were fixed in $80 \%$ ice-cold ethanol overnight at $-20^{\circ} \mathrm{C}$ and then incubated in $500 \mathrm{ml} \mathrm{PBS}$ containing $50 \mathrm{~g} / \mathrm{ml}$ propidium iodide and $20 \mu \mathrm{g} / \mathrm{ml}$ RNase for $30 \mathrm{~min}$. The data were analyzed using CellQuest version 3.3 software (BD Biosciences). The experiments were repeated three times.

MTT assays for cell viability. The cytotoxic effect of PTX was determined using MTT assays. Briefly, SKOV3 cells were cultured in 96-well plates at at a density of $5 \times 10^{3}$ cells/well and then treated with various concentrations of PTX $(1,30,90,300$, 1,000 or 3,000 nM; Sigma-Aldrich; Merck Millipore) for $48 \mathrm{~h}$. Subsequently, $20 \mathrm{ml}$ MTT (5 mg/ml; Sigma-Aldrich; Merck Millipore) was added to the wells, followed by incubation at $37^{\circ} \mathrm{C}$ for $4 \mathrm{~h}$. The supernatants were then aspirated, $100 \mathrm{ml}$ DMSO was added to the wells and the cells were incubated at $37^{\circ} \mathrm{C}$ for an additional $20 \mathrm{~min}$. The absorbance of the wells was then read using a microplate reader at a test wavelength of $570 \mathrm{~nm}$ and a reference wavelength of $630 \mathrm{~nm}$. Appropriate controls that lacked the cells were included to determine the background absorbance. The response to PTX treatment was assessed by standardizing the responses of the treatment groups to that of an untreated control.

Statistical analysis. All experiments were repeated at least three times, and the data are expressed as the mean \pm standard error. Statistical analyses were performed using SPSS 13.0 software for Windows and included Student's t-tests or one-way analysis of variance followed by Least Significant Difference and or Student-Newman-Keuls tests. $\mathrm{P}<0.05$ was considered statistically significant.

\section{Results}

Strategies for constructing single-site and multi-site shRNA vectors. In the present study, a platform to construct multi-shRNA vectors targeting a single gene was designed. The modified $\mathrm{p}$-EGFP-C1 vector containing three human U6 promoters was used as the blank vector. One target site (annealed forward and reverse oligonucleotides) was inserted after each U6 promoter. The selected target oligonucleotides annealed to the 5'-end, 3'-end or to the middle of the full-length DsRed gene (Fig. 1A). One shRNA was inserted after each of the U6 promoters (Fig. 1B). This process is much safer and easier than constructing lentiviral shRNA vectors. PCR was used to obtain the target fragment and an empty plasmid was selected as the blank vector. This method avoided the requirement for lentivirus packaging, reduced the mutation rate during PCR and permitted the same efficiency of silencing as when using lentiviral shRNA vectors.

The construction process for the three single-site shRNA vectors is shown in Fig. 1C. PCR was used to obtain a 267-bp shRNA fragment containing a promoter sequence, a target sequence, a loop sequence, a complementary sequence, a termination signal and a cloning site (Fig. 1D). The PCR products (Fig. 1E) that had been digested with Eco RI and Mlu I were ligated into the non-modified p-EGFP-C1 vectors.

For each shRNA, stem sequences matching a 21-base region of the target transcript, with an intervening 6-base 'loop', and which contained the corresponding cloning site, were designed. 
Table I. Sequences of oligonucleotides encoding the human DsRed-3shRNAs, with different flanking ends.

shRNA name

Oligonucleotide sequence

shRNA1 sense 5'-GATCCGGAGTTCATGCGCTTCAAGTTCAAGAGACTTGAAGCGCATGAACTCCTTTTTTCTGCA-3'

shRNA1 antisense 5'-GAAAAAAGGAGTTCATGCGCTTCAAGTCTCTTGAACTTGAAGCGCATGAACTCCG-3'

shRNA2 sense 5'-CGTTCATCGGCGTGAACTTCTTCAAGAGAGAAGTTCACGCCGATGAACTTTTTTGGTAC-3'

shRNA2 antisense 5'-CAAAAAAGTTCATCGGCGTGAACTTCTCTCTTGAAGAAGTTCACGCCGATGAACGAGCT-3'

shRNA3 sense 5'-AGCTTGTCCATCTACATGGCCAAGTTCAAGAGACTTGGCCATGTAGATGGACTTTTTTA-3'

shRNA3 antisense 5'-CGCGTAAAAAAGTCCATCTACATGGCCAAGTCTCTTGAACTTGGCCATGTAGATGGACA-3'

shRNA, short hairpin RNA.

A

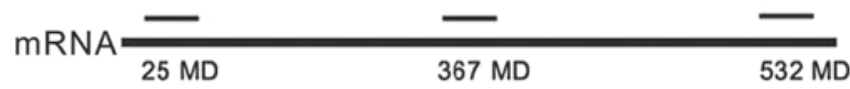

B
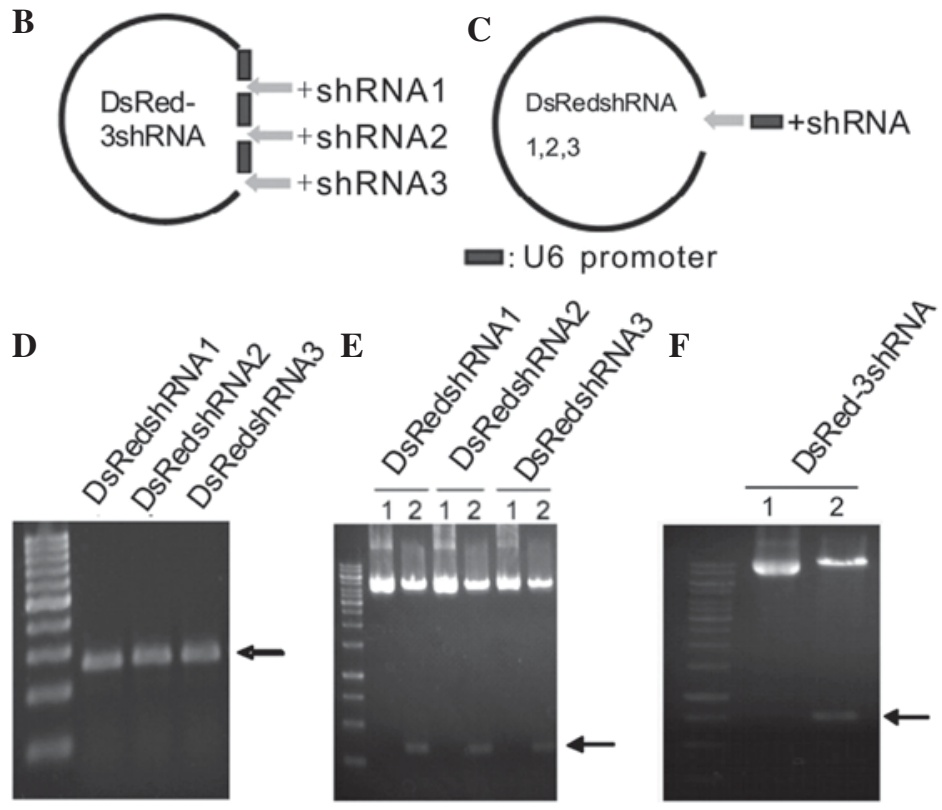

Figure 1. Construction of the multi-site shRNA vector and single-site shRNA vectors targeting the DsRed gene. The multi-site shRNA vector contained three DNA templates for the synthesis of shRNAs targeting the DsRed gene under the control of three U6 promoters. (A) Target sites of the three shRNAs in the full-length DsRed RNA transcript. (B) Map of the multi-site shRNA vector. Three human U6 promoter cassettes with different restriction sites were cloned from the pEGFP-C1 and pSIREN-DNR-DsRed-Express vectors by PCR and inserted into the pEGFP-C1 vector at Eco RI and Bam HI sites, Bam HI and Sac I sites, or Sac I and Hind III sites. (C) Map of the single-site shRNA vectors. The human U6 promter and one shRNA fragment were cloned from the full-length DsRed plasmid by PCR and inserted into pEGFP-C1 at Eco RI and Mlu I sites. (D) PCR products of human U6 promoters and single-shRNA fragments. (E and F) Positive clones were digested by double enzymes. Insert fragments were (E) 267 bp for single-site shRNA vectors and (F) 800 bp for the multi-site shRNA vector. shRNA, short hairpin RNA; PCR, polymerase chain reaction; EGFP, enhanced green fluorescent protein.

Multi-shRNA vector has a higher gene silencing efficiency than single-shRNA vectors for exogenous DsRed expression in HEK293 cells. The abilities of the multi-shRNA vector and three single-shRNA vectors to silence the DsRed exogenous gene in HEK293 cells was examined. Following establishment of the stable HEK293/DsRed cell line (Fig. 2A), four shRNA vectors, DsRed-3shRNA, DsRedshRNA1, DsRedshRNA2 and DsRedshRNA3, were transfected into the HEK293/DsRed cells. The RNAi plasmid p-EGFP-C1, which contained no target sequence, was used as a control.

A conventional optical fluoroscope was used to ensure that the DsRed plasmid was transfected into HEK293 cells, and it was observed that $>90 \%$ of the cells were red. Flow cytometry was then used to determine the percentage of red
HEK293/DsRed cells (Fig. 2A). Several methods were used to examine the RNAi effects, and the RNAi effects were observed at multiple time points in order to avoid the effect of time on the plasmid transfection efficiency. As shown in Fig. 2B-D, three points of time were analyzed and, at these points, fluorescent images were obtained using a camera and a conventional optical fluoroscope (Fig. 2B). Simultaneously, flow cytometric analysis was performed to calculate the intensity of red fluorescence and evaluate its attenuation, which indirectly corresponded to the extent of DsRed gene silencing at the protein level (Fig. 2C). Using RT-qPCR, the ability of the different shRNA vectors to silence the mRNA expression of the DsRed gene was assessed at different time points following transfection (Fig. 2D). Finally, using semiquantitative RT-PCR, 
Table II. Primers for DsRed single-shRNA vectors.

\begin{tabular}{lcc}
\hline Vectors & Forward primers & \multicolumn{1}{c}{ Reverse primers } \\
\hline DsRedshRNA1 & 5'-GGAATTCAAGCGGCCGCATAACTTCG-3' & 5'-CGACGCGTAAAAAAGGAGTTCATGCGC \\
& & TTCAAGTCTCTTGAACTTGAAGCGCATGA \\
DsRedshRNA2 & 5'-GGAATTCAAGCGGCCGCATAACTTCG-3' & 5'-CGACGGTCCTTTCCACAAGA-3' \\
& & AACTTCTCTCTTGAAGAAGTTCACGCCGA \\
DsRedshRNA3 & 5'-GGAATTCAAGCGGCCGCATAACTTCG-3' & TGAACTCGTCCTTTCCACAAGA-3' \\
& & 5'-CGACGCGTAAAAAAGTCCATCTACATGG \\
& & CCAAGTCTCTTGAACTTGGCCATGTAGATG \\
& GACTCGTCCTTTCCACAAGA-3'
\end{tabular}

shRNA, short hairpin RNA.

Table III. Primer sequences for reverse transcription-quantitative polymerase chain reaction.

\begin{tabular}{lll}
\hline Name & \multicolumn{1}{c}{ Forward primers } & \multicolumn{1}{c}{ Reverse primers } \\
\hline DsRed & 5'-CGGTCTGGGTGCCCTCGTAG-3' & 5'-CAAGGAGTTCATGCGCTTCA-3' \\
GFP & 5'-GGATCCCGCCACCATGGTGAGCAAG-3' & 5'-GAATTCCTTGTACAGCTCGTCCATG-3' \\
GAPDH & 5'-AGAAGGCTGGGGCTCATTTA-3' & 5'-AGGGGCCATCCACAGTCTTC-3' \\
OAS1 & 5'-TCAGAAGAGAAGCCAACGTGA-3' & 5-CGGAGACAGCGAGGGTAAAT-3' \\
AKT1 & 5'-GTGGACCAACGTGAGGCTC-3' & 5'-GAAGGTGCGTTCGATGACAG-3' \\
AKT2 & 5'-CAAGCGTGGTGAATACATCAAGA-3' & 5'-GCCTCTCCTTGTACCCAATGA-3' \\
AKT3 & 5'-TCTTACACATAGCAGGGGCACCTTC-3' & 5'-CAGTAGCAGCAACAGCATGAGACC-3' \\
\hline
\end{tabular}

GFP, green fluorescent protein; OAS1, 2'-5'-oligoadenylate synthase 1 .

the relative expression levels of DsRed in cells transfected with two shRNAs (i.e., co-transfected with two single-shRNA vectors), the DsRed-3shRNA vector or a single-shRNA vector were determined (Fig. 2E). GFP was the tag protein used to confirm the successful transfection of the shRNA vectors.

After 2 days of transfection, it was evident that the multi-shRNA vector produced stronger RNAi effects compared with the single-shRNA vector $(\mathrm{P}<0.05)$ and the two-shRNA vector $(\mathrm{P}<0.01)$ at the mRNA and protein expression levels. However, within the first 2 days, there was no obvious difference in the fluorescence intensity of the cells transfected with different shRNA vectors. Additionally, within the first 2 days, the expression of DsRed did not change significantly following transfection with the different shRNA vectors at the mRNA and protein levels $(\mathrm{P}>0.05)$.

IFN response assay to verify the specificity of $R N A$ interference. In most mammalian cells, the use of long ( $>30$ nucleotides) double-stranded (ds)RNA provokes an interferon (IFN) response; in particular, a non-specific type 1 IFN response that can lead to nonspecific gene suppression and a general shutdown of protein synthesis (20). Using our RNAi library, we designed multi-shRNA vectors that formed 19-bp dsRNAs in cells. However, the specificity of the RNAi had to be verified. According to Jaitin and Schreiber (21), if IFN signaling pathways are induced, the expression of 2'-5'-oligoadenylate synthase 1
(OAS1) at the mRNA level and STAT1 at the protein level are enhanced. In the present study, $2 \mu$ l exogenous IFN- $\beta$ was added to HEK293/DsRed cells transfected with DsRedshRNA1, DsRedshRNA2, DsRedshRNA3 or DsRed-3shRNA for $>16 \mathrm{~h}$ to induce the IFN signaling pathways. If an endogenous IFN response occurred, the expression of OAS1 mRNA and STAT1 protein should not alter considerably following the addition of exogenous IFN- $\beta$. However, a marked increase in the expression of OAS1 mRNA (Fig. 3A) and STAT1 protein (Fig. 3B) were observed following the addition of exogenous IFN- $\beta$ $(\mathrm{P}<0.01)$, which suggested that an exogenous, but not an endogenous, IFN response was generated in the shRNA-transfected HEK293/DsRed cells. Therefore, the gene silencing effects that were induced by the different shRNA vectors were specific.

Multi-shRNA vector silences the expression of the endogenous Akt2 gene in SKOV3 cells more effectively than single-and two-site shRNA vectors. The endogenous gene, Akt2, was selected as the target because, in our previous studies, it was shown that Akt2 was highly expressed in the SKOV3 human ovarian cancer cell line $(15,16)$. First, images of SKOV3 cells transfected with the Akt2shRNA, Akt2-2shRNA, Akt2-3shRNA or negative control vectors were obtained using a conventional optical fluoroscope to determine their transfection efficiencies (Fig. 4A). Subsequently, the mRNA and protein expression of Akt 2 in the shRNA-transfected cells 

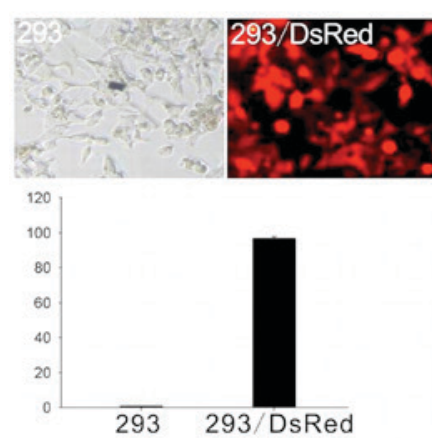

B

$2 d$

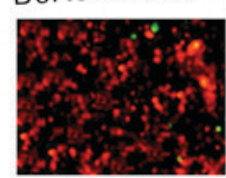

$4 d$

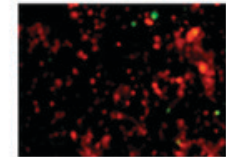

$7 d$

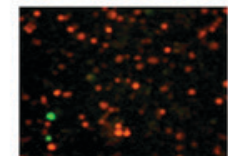

C

$\mathbf{E}$

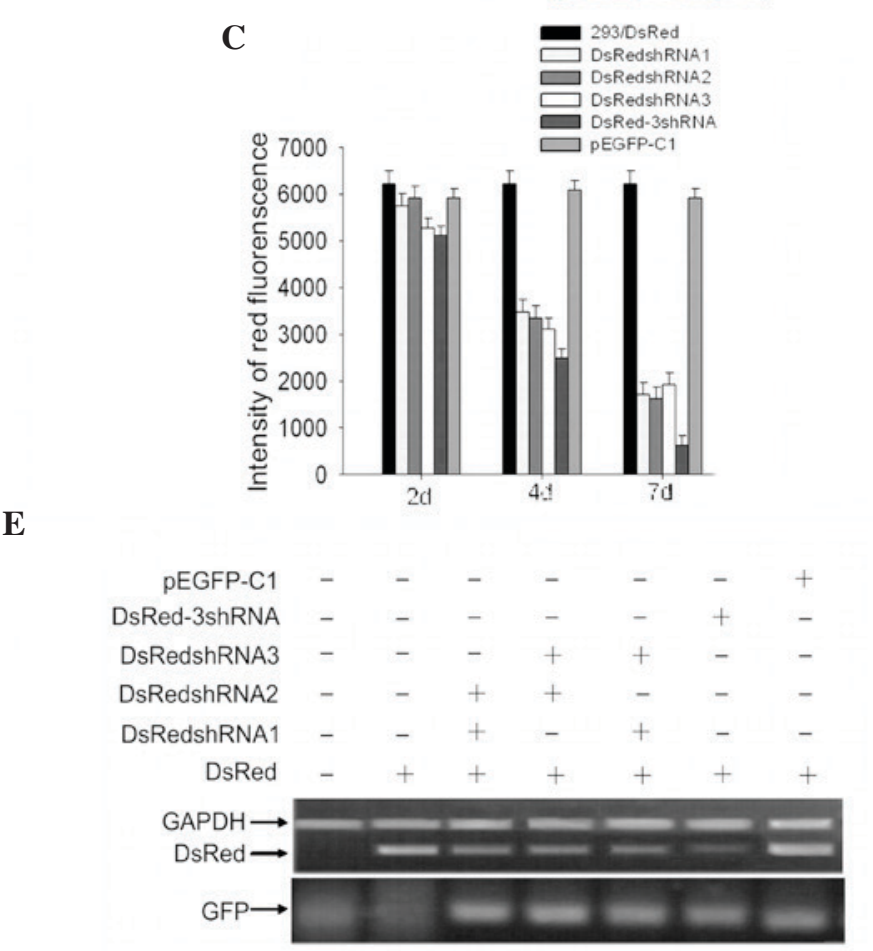

1 DsRedshRNA2
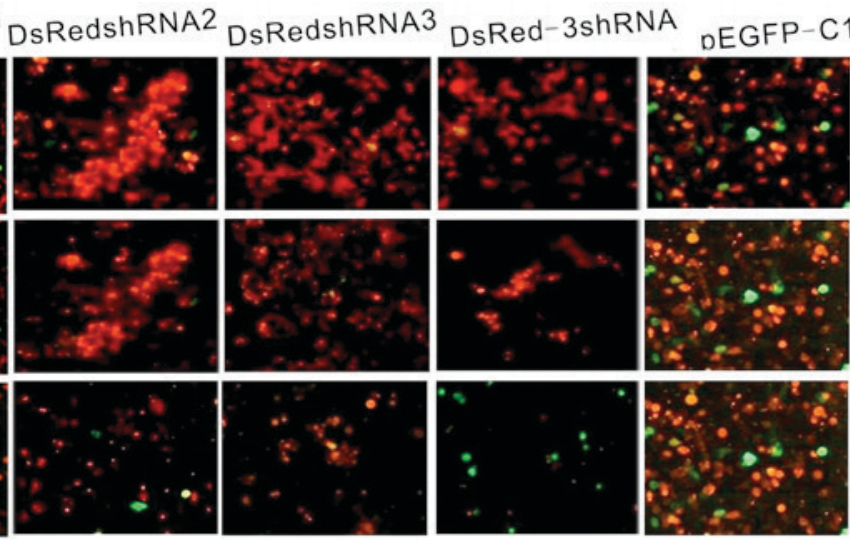

D
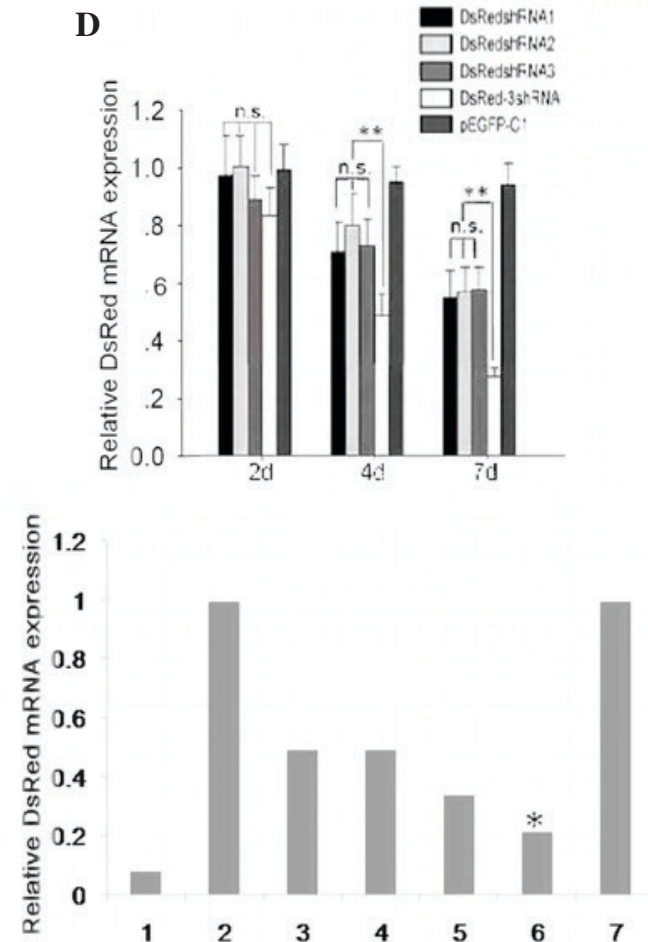

Figure 2. DsRed gene silencing in the HEK293/DsRed cell line using DsRed-3shRNA, DsRedshRNA1, DsRedshRNA2 and/or DsRedshRNA3 vectors (A) Stable expression of DsRed in the HEK293 cell line was confirmed by fluorescent microscopy and flow cytometry, which demonstrated that $>90 \%$ of the cells were emitting red fluorescence. (B) The fluorescence of the cells at 4 and 7 days following transfection with the vectors was assessed using a fluorescence microscope. (C) Red fluorescence analysis by fluorescence-activated cell sorting and (D) RT-qPCR demonstrated that the DsRed-3shRNA vector downregulated the expression of DsRed to a greater extent after 4 and 7 days than DsRedshRNA1, 2 or 3 vectors $\left({ }^{* *} \mathrm{P}<0.01\right)$. There was no statistical significance between the four vectors and the empty vector after 2 days. Values from at least three-independent experiments were pooled and expressed as the mean \pm standard error (E) RT-PCR showed that the DsRed-3shRNA vector exerted better silencing effects on the DsRed gene in 293/DsRed cells than any other combination of the DsRedshRNA1, DsRedshRNA2 and DsRedshRNA3 vectors after 7 days ( $\left.{ }^{*}<0.05\right)$. shRNA, short hairpin RNA; RT-qPCR, reverse transcription-quantitative polymerase chain reaction; RT-PCR, reverse transcription-polymerase chain reaction; GFP, green fluorescent protein.

was detected (Fig. 4B and C). Notably, the Akt2-3shRNA vector had a stronger silencing effect than the Akt2shRNA or Akt2-2shRNA vectors, as compared with the control $(\mathrm{P}<0.05)$; however, the Akt2shRNA and Akt2-2shRNA vectors had stronger silencing effects than the control $(\mathrm{P}<0.05)$.

RT-qPCR was used to determine the gene silencing specificity (Fig. 5). It was found that, in all shRNA vector-transfected cells, the mRNA expression levels of Akt1 and Akt3 did not change significantly $(\mathrm{P}>0.05)$, while Akt2 was silenced. In addition, it was observed that the single-site shRNA vectors produced more frequent off-target effects than the two-site and multi-site shRNA vectors ( $\mathrm{P}<0.05$; Fig. 5). Notably, the same results were obtained when other genes were silenced (data not shown).
Multi-site shRNA vector shows stronger loss-of-function of the endogenous Akt2 gene in SKOV3 cells than the single-shRNA and two-site shRNA vectors. As shown in Fig. 6, the drug tolerance of SKOV3 cells to PTX following silencing of the endogenous Akt2 gene with the different vectors was detected. Following treatment of the shRNA vector-transfected SKOV3 cells with various concentrations of PTX for $48 \mathrm{~h}$, MTT assays were performed to detect the cell viability. According to the data, cells transfected with the Akt2-3shRNA vector showed a markedly lower viability than the other cells (Fig. 6A). The half maximal inhibitory concentration values were increased 5.16and 3.57-fold in cells transfected with the Akt2-shRNA and Akt2-2shRNA vectors, respectively $(\mathrm{P}<0.01)$, and 17.24- and 21.07-fold in the negative control and blank control cells, 

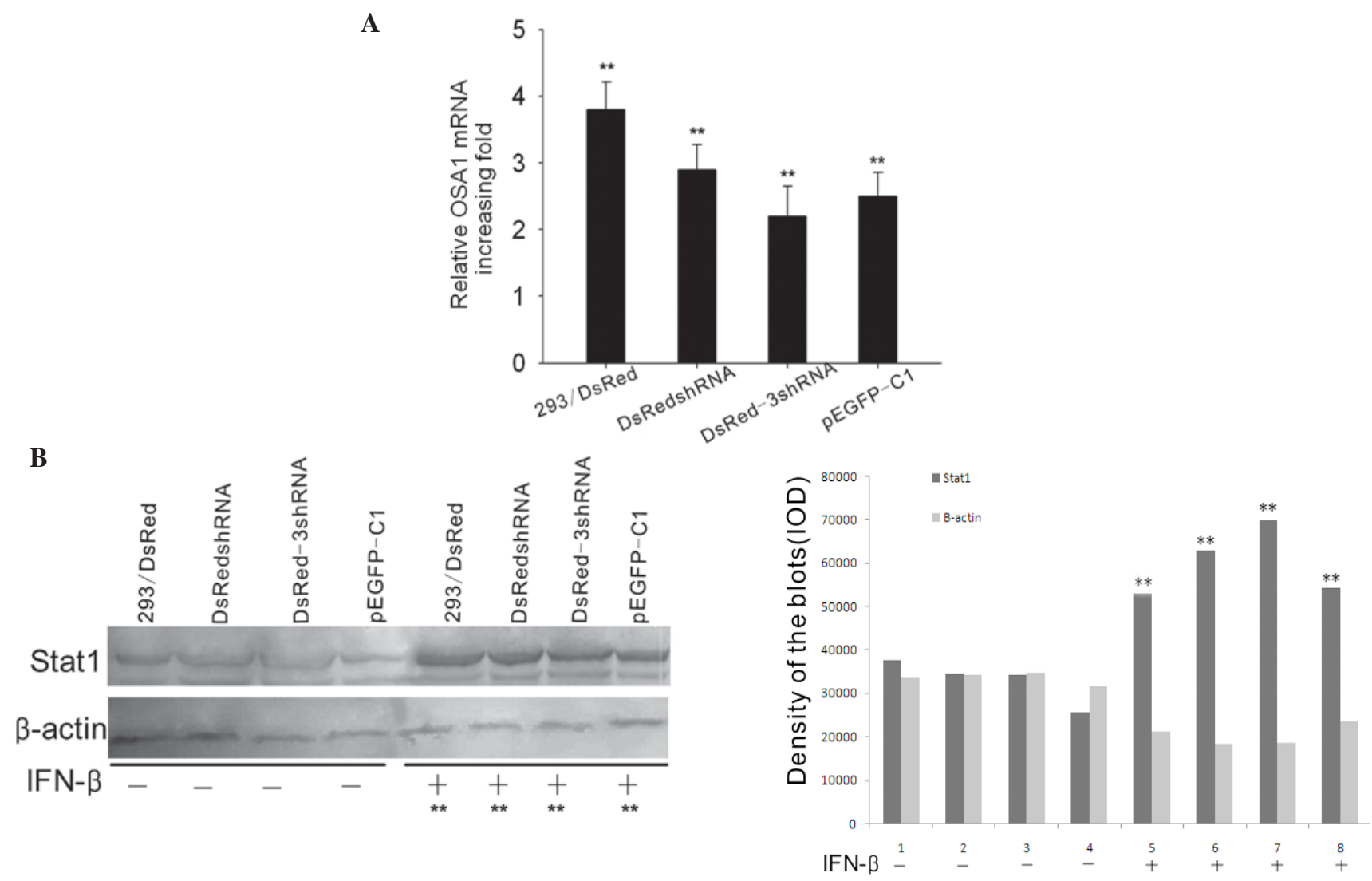

Figure 3. IFN response assay. The HEK293/DsRed cell line was transfected with various shRNA vectors and treated with IFN- $\beta$ for 16 h. (A) Semiquantitative reverse transcription-polymerase chain reaction was performed to measure the mRNA expression level of OAS1. The mRNA expression of OAS1 increased 3.8 \pm 0.51-, 2.8 $\pm 0.34-, 2.3 \pm 0.23$ - and 2.7 \pm 0.31 -fold in 293/DsRed, 293/DsRed/DsRedshRNA, 293/DsRed/DsRed-3shRNA and 293/DsRed/pEGFP-C1 cells, respectively, when IFN- $\beta$ was added for $16 \mathrm{~h}$. The bar graph shows the results of three independent experiments. ${ }^{* *} \mathrm{P}<0.01$. (B) The protein expression of STAT1 was increased 2.26-, 3.43-, 3.85- and 2.83-fold in the 293/DsRed, 293/DsRed/DsRedshRNA, 293/DsRed/DsRed-3shRNA and 293/DsRed/pEGFP-C1 cells, respectively, when IFN- $\beta$ was added for $16 \mathrm{~h}$, as determined by western blotting "** $\mathrm{P}<0.01$. IFN, interferon; shRNA, short hairpin RNA; OSA1, 2'-5'-oligoadenylate synthase 1; STAT1, signal transducer and activator of transcription.

respectively $(\mathrm{P}<0.01)$, as compared with the cells transfected with the Akt2-3shRNA vector.

The apoptosis rate was evaluated following treatment with $90 \mathrm{nM}$ PTX for $48 \mathrm{~h}$ (Fig. 6B). Cells transfected with the Akt2-3shRNA vector displayed an apoptosis rate of $26 \pm 2.32 \%$, which was significantly higher than that of other cells, including an apoptotic rate of $18 \pm 3.14 \%$ in SKOV3 cells transfected with Akt2-2shRNA $(\mathrm{P}=0.02)$ and that of $15 \pm 2.06 \%$ in SKOV3 cells transfected with Akt2-shRNA $(\mathrm{P}<0.001)$. The apoptosis rates of the cells transfected with the three shRNA vectors were all higher than those of the negative $(6 \pm 1.92 \%)$ or blank controls $(5 \pm 1.41 \%)$, and these differences were statistically significant $(\mathrm{P}<0.01)$.

\section{Discussion}

The application of RNAi has revolutionized the study of gene function in vitro and promises to facilitate large-scale loss-of-function studies in human cells (22). Recently, siRNA and shRNA vectors have been used successfully (23), but many practical and theoretical problems remain before RNAi becomes routine. To develop a resource that will enable broad and efficient silencing in human cells, the present study designed a platform for the construction of multi-site shRNA vectors that contain three shRNA fragments targeting different sites of a single gene. To verify the effectiveness of this method, the exogenous reporter gene DsRed and the endogenous gene Akt 2 were selected as the two target genes and their corresponding multiple and single RNAi vectors were constructed. The present study demonstrated that the multi-site shRNA vector had better silencing effects on DsRed at the mRNA and protein expression levels than the single-site and two-site vectors after 2 days, although no significant differences were observed before that time. In addition, the multi-site shRNA vector exhibited the best loss-of-function effects on the endogenous Akt2 gene, as it had the most powerful ability to reverse PTX-induced resistance in SKOV3 cells. Furthermore, the IFN response to the shRNA vectors was assessed, according to a previous study (21). Notably, the mRNA expression of OAS1 and the protein expression of STAT1 were not readily detected following addition of exogenous IFN- $\beta$ to the cells. In addition, when Akt 2 was silenced using the various shRNA vectors, the expression levels of its homologous genes, Akt1 and Akt3, did not change significantly $(\mathrm{P}>0.05)$, and were changed to the least extent in the cells transfected with the multi-site shRNA vector, suggesting that this vector decreased the occurrence of off-target effects.

Currently, the most popular shRNA technique involves a single shRNA ligated into a plasmid or lentiviral vector containing polymerase III promoters $(24,25)$. Transfection of 
A

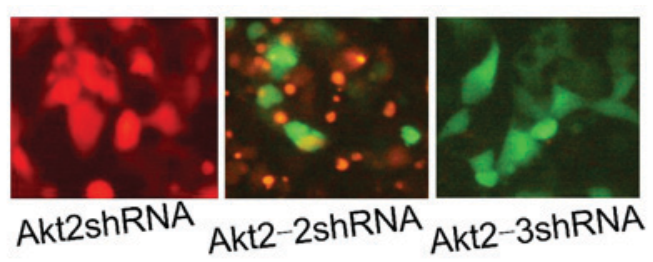

B
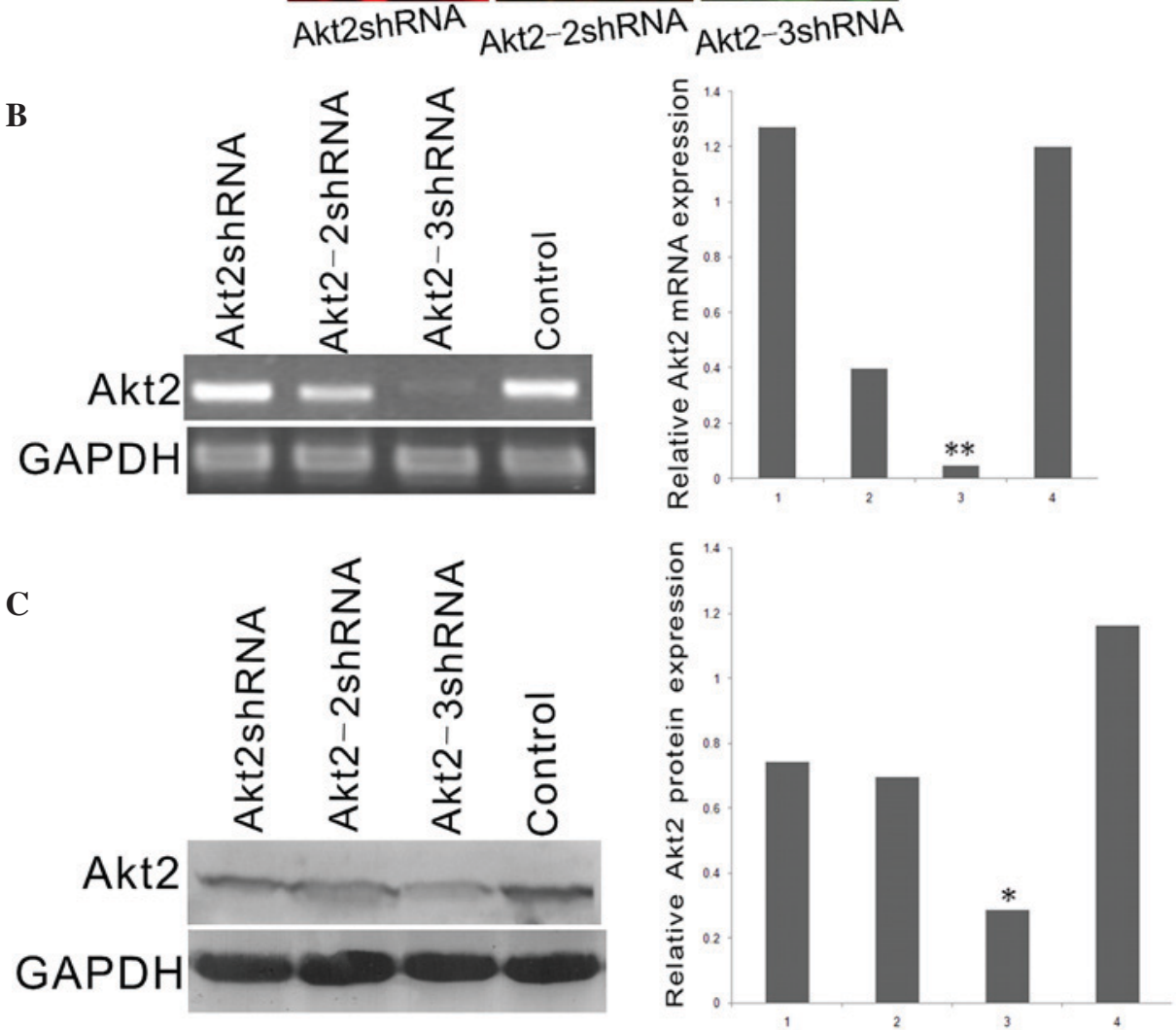

Figure 4. Akt2 gene silencing in SKOV3 cells by Akt2shRNA1, Akt2-2shRNA or Akt2-3shRNA vectors. (A) Images of SKOV3 cells transfected with Akt2shRNA, Akt2-2shRNA and Akt2-3shRNA vectors were obtained using a fluorescent microscope to assess the transfection efficiency. (B) Reverse transcription-polymerase chain reaction and $(\mathrm{C})$ western blotting demonstrated that the Akt2-3shRNA vector downregulated Akt2 expression in SKOV3 cells to a greater extent than Akt2shRNA, Akt2-2shRNA and control vectors at the mRNA and protein levels after 4 days $\left({ }^{*} \mathrm{P}<0.05,{ }^{* *} \mathrm{P}<0.01\right)$. shRNA, small hairpin RNA.

cells with the plasmid or lentiviral vector can then eliminate the expression of a target gene $(26,27)$. However, off-target effects, which are the result of partial homology to other transcripts, complicate the application of shRNAs $(28,29)$. To avoid this inherent property of shRNAs, numerous study groups have screened for the optimum shRNA $(5,30)$. In our previous study, two independent, 19-nucleotide sequences that targeted the Akt2 gene were designed and were cloned into two different plasmids (15). Subsequently, SKOV3 cells were co-transfected with the two shRNA vectors and it was demonstrated that the two-site shRNA reduced the expression of the Akt2 gene more effectively than the single-site shRNA (15). However, co-transfection is not always convenient because many mammalian cells are not easily transfected, and many cells exhibit plasmid incompatibility (31). To mitigate these problems, Berns et al (11) proposed the concept of multi-site shRNAs for large-scale RNAi screens in human cells. Other groups have constructed multiple shRNA vectors to knockdown multiple genes (32-34), and many groups have constructed lentiviral shRNA vectors when establishing an RNAi library $(5,35)$. These improvements resulted in on-target effects by the shRNA vectors on the corresponding genes. Therefore, multi-site shRNA plasmids are popular and have been widely applied in human cells $(36,37)$; however, few studies have investigated the properties of multi-site shRNA vectors targeting a single gene.

The present study compared the suppression abilities of different-site shRNA vectors. Previously, endogenous genes have always been assessed (36); thus, the present study included the exogenous reporter gene DsRed and an endogenous gene as targets. First, the construction methods of multi-site shRNA vectors were demonstrated, which involved reconstructing the pEGFP-C1 into the pEGFP-C1-U6-U6-U6 vector, which carries three human U6 promoters. The shRNA double oligonucleotides were synthesized in vitro, annealed and ligated into the reconstructed vector to form the multi-site RNAi vector. This strategy bypassed the mutation rate problem of PCR and, therefore, further simplified the construction procedure.

After the construct was successfully made, whether the multi-site shRNA vector was more advantageous than the single-site and two-site shRNA vectors for silencing an exogenous gene in vitro was investigated. Since few studies have performed silencing of exogenous genes in mammalian cells, the post-inhibition time points were broadened to ensure that the silencing effects of the various shRNA vectors were observed. The results demonstrated that the multi-site shRNA 
A

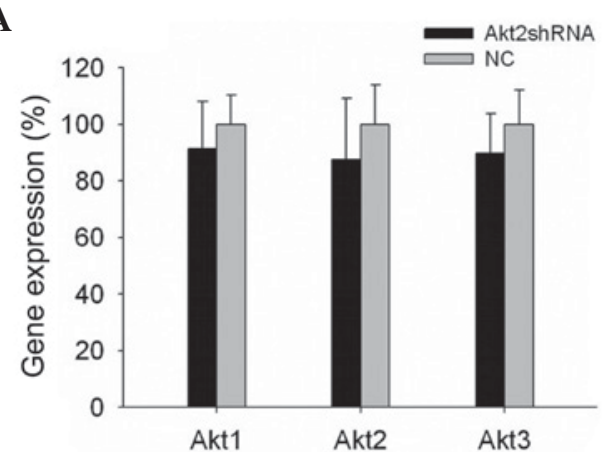

B
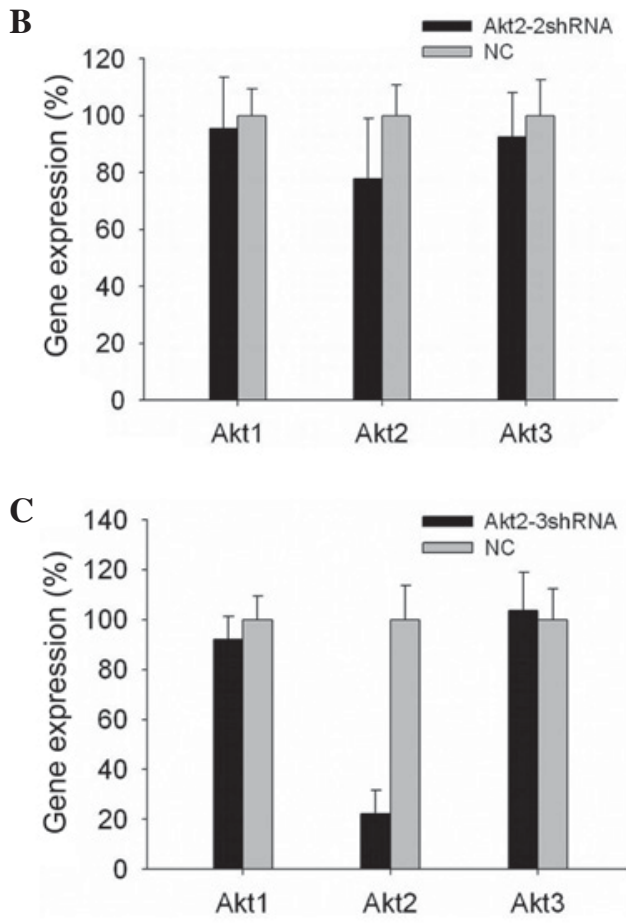

Figure 5. RNA interfence specificity assay. Semiquantitative reverse transcription-polymerase chain reaction was performed to measure the mRNA expression levels of Akt1, Akt 2 and Akt 3 in SKOV3 cells transfected with various shRNA vectors. The expression of Akt 2 was decreased to (A) $87 \pm 24.9 \%$, (B) $77 \pm 23.2 \%$ and (C) $21 \pm 8.15 \%$ in SKOV3 cells transfected with Akt2shRNA, Akt2-2shRNA and Akt2-3shRNA, respectively. The differences between the cells transfected with Akt2shRNA and Akt2-2shRNA and those transfected with Akt2-2shRNA and Akt2-3shRNA were significantly different $(\mathrm{P}<0.05)$. Values from at least three-independent experiments were pooled and expressed as the mean \pm standard error. shRNA, small hairpin RNA.

vector could maintain a longer and higher RNAi effect than the single-site and two-site shRNA vectors.

When targeting the endogenous Akt2 gene, it was determined that the 2-day time point was the optimum time point for observing the effects. Within 2 days, the multi-site shRNA vector had suppressed the expression of Akt2 at the mRNA and protein levels and exhibited a significantly greater loss-of-function than the single-site and two-site shRNA vectors. The off-target effects of the vectors were assessed by detecting the expression of genes that were homologous to Akt2. While the expression levels of Akt1 and Akt3 decreased marginally in the cells transfected with the single-site shRNA, the expression levels of these genes were unchanged in the two-site and multi-site groups. These results suggested that
A

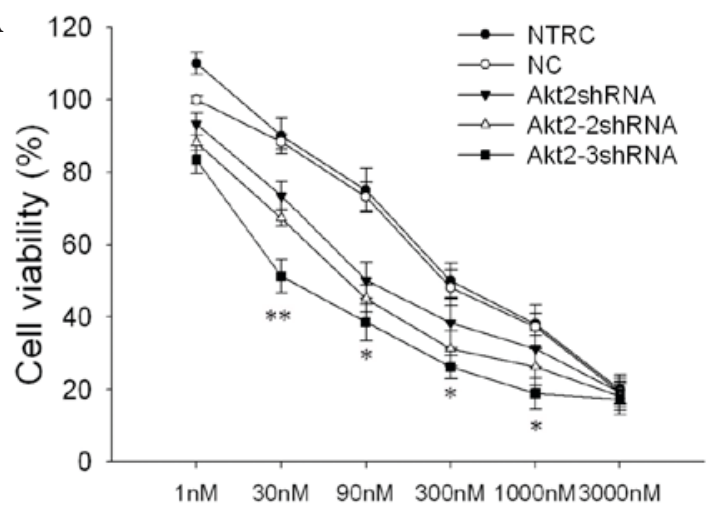

B

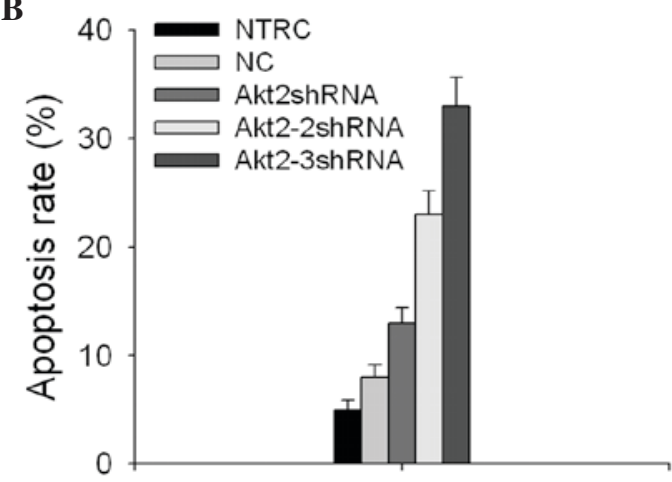

Figure 6. Inhibition of Akt2 expression using the Akt2-3shRNA vector enhanced PTX-induced SKOV3 cell death to a greater extent than Akt2shRNA and Akt2-2shRNA vectors. (A) Effects of Akt2 suppression on sensitizing SKOV3 cells to PTX treatment. Cells were exposed to gradually increasing concentrations of PTX and incubated for $48 \mathrm{~h}$. Cell viability was determined using MTT assays. ${ }^{*} \mathrm{P}<0.05,{ }^{* *} \mathrm{P}<0.01$ vs. the other groups. (B) Apoptosis was determined by flow cytometric analysis using propidium iodide straining. Cells were treated with $90 \mathrm{nM}$ PTX for $48 \mathrm{~h}$ and then evaluated. The cells transfected with Akt2-3shRNA vector showed a higher apoptosis rate than the cells transfected with Akt2shRNA, Akt2-2shRNA and NC vectors. The graphs show the results of three independent experiments. PTX, paclitaxel; NC, normal control; shRNA, short hairpin RNA; NTRC, non-transfected cells.

multi-site shRNA interference could simultaneously enhance suppression efficiency and reduce off-target effects.

Various limitations associated with RNAi still exist, even though steps toward improvement were made in this study. The present study did not performe research on cells from organisms other than humans and, therefore, it may not be assumed that the RNAi effects using this strategy are the same in other organisms as in human cells. Further studies of a braod array of cell types are required. Furthermore, although plasmids are safer tools than lentiviruses, when using suspended cells, such as blood and stem cells, lentiviruses may be the better choice for silencing genes.

In summary, the present study produced a highly efficient, multi-site shRNA vector that targeted exogenous and endogenous genes in human cells. This method was much safer, easier to perform and more suitable for constructing an RNAi library, as compared with methods involving co-transfection of cells with two or three shRNAs to silence a single gene. In addition, the time requirement was reduced by not having to screen for the best shRNA and position problems were avoided during the process of construction. These accomplishments will likely broaden the application of these novel vectors 
for studying gene function in vivo. Furthermore, a specific advantage was the possibility of targeting three different genes simultaneously, which overcomes the difficulty of co-transfection. Using this system, the function of more than one gene can be monitored and controlled without the risk of off-target effects. The multi-site shRNA vector that is described here is particularly beneficial when searching for a wide range of novel genes and is valuable for evaluating signaling pathways involved in tumorigenesis and the mechanisms of chemoresistance, as well as the exploitation of adenovirus restructuring drugs.

\section{Acknowledgements}

This study was supported by grants from the National Natural Science Foundation of China (nos. 30973184 and 81101971), Guangdong Natural Science Foundation (nos. B2011295 and S2011040006012) and the '973' Program of China (no. 2009CB521800).

\section{References}

1. Fire A,Xu S,Montgomery MK, Kostas SA,DriverSEand Mello CC: Potent and specific genetic interference by double-stranded RNA in Caenorhabditis elegans. Nature 391: 806-811, 1998.

2. Elbashir SM, Harborth J, Lendeckel W, Yalcin A, Weber K and Tuschl T: Duplexes of 21-nucleotide RNAs mediate RNA interference in cultured mammalian cells. Nature 411: 494-498, 2001

3. Crombez L and Divita G: A non-covalent peptide-based strategy for siRNA delivery. Methods Mol Biol 683: 349-360, 2011.

4. Shan G, Li Y, Zhang J, Li W, Szulwach KE, Duan R, Faghihi MA, Khalil AM, Lu L, Paroo Z, et al: A small molecule enhances RNA interference and promotes microRNA processing. Nat Biotechnol 26: 933-940, 2008.

5. Moffat J, Grueneberg DA, Yang X, Kim SY, Kloepfer AM, Hinkle G, Piqani B, Eisenhaure TM, Luo B, Grenier JK, et al: A lentiviral RNAi library for human and mouse genes applied to an arrayed viral high-content screen. Cell 124: 1283-1298, 2006

6. Ku SH, Jo SD, Lee YK, Kim K and Kim SH: Chemical and structural modifications of RNAi therapeutics. Adv Drug Deliv Rev 104: 16-28, 2016.

7. Khandelwal N, Breinig M, Speck T, Michels T, Kreutzer C, Sorrentino A, Sharma AK, Umansky L, Conrad H, Poschke I, etal: A high-throughput RNAi screen for detection of immune-checkpoint molecules that mediate tumor resistance to cytotoxic T lymphocytes. EMBO Mol Med 7: 450-463, 2015.

8. Cho JS, Kim YC and Morrison SL:Inhibitors of MyD88-dependent proinflammatory cytokine production identified utilizing a novel RNA interference screening approach. PLoS One 4: e7029, 2009.

9. Du C, Ge B, Liu Z, Fu K, Chan WC and McKeithan TW: PCR-based generation of shRNA libraries from cDNAs. BMC Biotechnol 6: 28, 2006.

10. Scherer LJ, Yildiz Y, Kim J, Cagnon L, Heale B and Rossi JJ: Rapid assessment of anti-HIV siRNA efficacy using PCR-derived pol III shRNA cassettes. Mol Ther 10: 597-603, 2004.

11. Berns K, Hijmans EM, Mullenders J, Brummelkamp TR, Velds A, Heimerikx M, Kerkhoven RM, Madiredjo M, Nijkamp W, Weigelt B, et al: A large-scale RNAi screen in human cells identifies new components of the p53 pathway. Nature 428: 431-437, 2004.

12. Stewart SA, Dykxhoorn DM, Palliser D, Mizuno H, Yu EY, An DS, Sabatini DM, Chen IS, Hahn WC, Sharp PA, et al: Lentivirus-delivered stable gene silencing by RNAi in primary cells. RNA 9: 493-501, 2003.

13. Klinghoffer RA, Roberts B, Annis J, Frazier J, Lewis P, Linsley PS and Cleary MA: An optimized lentivirus-mediated RNAi screen reveals kinase modulators of kinesin-5 inhibitor sensitivity. Assay Drug Dev Technol 6: 105-119, 2008.

14. Wang YH, Wang ZX, Qiu Y, Xiong J, Chen YX, Miao DS and De W: Lentivirus-mediated RNAi knockdown of insulin-like growth factor-1 receptor inhibits growth, reduces invasion, and enhances radiosensitivity in human osteosarcoma cells. Mol Cell Biochem 327: 257-266, 2009.
15. Xing H, Weng D, Chen G, Tao W, Zhu T, Yang X, Meng L, Wang S, Lu Y and Ma D: Activation of fibronectin/PI-3K/Akt2 leads to chemoresistance to docetaxel by regulating survivin protein expression in ovarian and breast cancer cells. Cancer Lett 261: 108-119, 2008.

16. Weng D, Song X, Xing H, Ma X, Xia X, Weng Y, Zhou J, Xu G, Meng L, Zhu T, et al: Implication of the Akt2/survivin pathway as a critical target in paclitaxel treatment in human ovarian cancer cells. Cancer Lett 273: 257-265, 2009.

17. Manche L, Green SR, Schmedt C and Mathews MB: Interactions between double-stranded-RNA regulators and the protein-kinase DAI. Mol Cell Biol 12: 5238-5248, 1992.

18. Williams BR: Role of the double-stranded RNA-activated protein kinase $(\mathrm{PKR})$ in cell regulation. Biochem Soc Trans 25: 509-513, 1997.

19. Livak KJ and Schmittgen TD: Analysis of relative gene expression data using real-time quantitative PCR and the 2(-Delta Delta C(T)) Method. Methods 25: 402-408, 2001

20. Uprichard SL: The therapeutic potential of RNA interference. FEBS Lett 579: 5996-6007, 2005.

21. Jaitin DA and Schreiber G: Upregulation of a small subset of genes drives type I interferon-induced antiviral memory. J Interferon Cytokine Res 27: 653-664, 2007.

22. Leung RK and Whittaker PA: RNA interference: From gene silencing to gene-specific therapeutics. Pharmacol Ther 107: 222-239, 2005.

23. Elbashir SM, Harborth J, Weber K and Tuschl T: Analysis of gene function in somatic mammalian cells using small interfering RNAs. Methods 26: 199-213, 2002.

24. Rubinson DA, Dillon CP, Kwiatkowski AV, Sievers C, Yang L, Kopinja J, Rooney DL, Zhang M, Ihrig MM, McManus MT, et al: A lentivirus-based system to functionally silence genes in primary mammalian cells, stem cells and transgenic mice by RNA interference. Nat Genet 33: 401-406, 2003.

25. Tiscornia G, Singer O and Verma IM: Design and cloning of lentiviral vectors expressing small interfering RNAs. Nat Protoc 1: 234-240, 2006.

26. Roelz R, Pilz IH, Mutschler M and Pahl HL: Of mice and men: Human RNA polymerase III promoter U6 is more efficient than its murine homologue for shRNA expression from a lentiviral vector in both human and murine progenitor cells. Exp Hematol 38: 792-797, 2010.

27. Miest T, Saenz D, Meehan A, Llano M and Poeschla EM: Intensive RNAi with lentiviral vectors in mammalian cells. Methods 47: 298-303, 2009.

28. Jackson AL, Burchard J, Leake D, Reynolds A, Schelter J, Guo J, Johnson JM, Lim L, Karpilow J, Nichols K, et al: Position-specific chemical modification of siRNAs reduces 'off-target' transcript silencing. RNA 12: 1197-1205, 2006

29. Sledz CA, Holko M, de Veer MJ, Silverman RH and Williams BR: Activation of the interferon system by short-interfering RNAs. Nat Cell Biol 5: 834-839, 2003.

30. Boudreau RL, Monteys AM and Davidson BL: Minimizing variables among hairpin-based RNAi vectors reveals the potency of shRNAs. RNA 14: 1834-1844, 2008.

31. Feinbaum R: Introduction to plasmid biology. Curr Protoc Mol Biol Chapter 1: Unit1.5, 2001.

32. Xia XG, Zhou H and Xu Z: Multiple shRNAs expressed by an inducible pol II promoter can knock down the expression of multiple target genes. Biotechniques 41: 64-68, 2006.

33. Stove V, Smits K, Naessens E, Plum J and Verhasselt B: Multiple gene knock-down by a single lentiviral vector expressing an array of short hairpin RNAs. Electron J Biotechnol 9: 572-579, 2006.

34. Xu XM, Yoo MH, Carlson BA, Gladyshev VN and Hatfield DL: Simultaneous knockdown of the expression of two genes using multiple shRNAs and subsequent knock-in of their expression. Nat Protoc 4: 1338-1348, 2009.

35. Root DE, Hacohen N, Hahn WC, Lander ES and Sabatini DM: Genome-scale loss-of-function screening with a lentiviral RNAi library. Nat Methods 3: 715-719, 2006.

36. Song J, Giang A, Lu Y, Pang S and Chiu R: Multiple shRNA expressing vector enhances efficiency of gene silencing. BMB Rep 41: 358-362, 2008.

37. Kim SM, Lee KN, Lee SJ, Ko YJ, Lee HS, Kweon CH, Kim HS and Park JH: Multiple shRNAs driven by U6 and CMV promoter enhances efficiency of antiviral effects against foot-and-mouth disease virus. Antiviral Res 87: 307-317, 2010. 